\title{
Genetic conversion of a split-drive into a full-drive element
}

\author{
Gerard Terradas ${ }^{1,2,6}$, Jared B. Bennett ${ }^{3}$, Zhiqian $\mathrm{Li}^{1,2}$, John M. Marshall ${ }^{4,5}$, Ethan Bier ${ }^{1,2^{*}}$
}

${ }^{1}$ Section of Cell and Developmental Biology, University of California, San Diego, La Jolla, CA 92093, USA

${ }^{2}$ Tata Institute for Genetics and Society, University of California, San Diego, La Jolla, CA 92093, USA

${ }^{3}$ Biophysics Graduate Group, Division of Biological Sciences, College of Letters and Science, University of California, Berkeley, CA 94720, USA

${ }^{4}$ Division of Epidemiology and Biostatistics, School of Public Health, University of California, Berkeley, CA 94720, USA

${ }^{5}$ Innovative Genomics Institute, Berkeley, CA 94720, USA

${ }^{6}$ Current address: Department of Entomology, The Center for Infectious Disease Dynamics, and the Huck Institutes for the Life Sciences, The Pennsylvania State University, University Park, PA 16801, USA

* Corresponding author

e-mail: ebier@ucsd.edu

Key words: split drive, full drive, Drosophila, Cas9, CRISPR, hacking, gene drive, active genetics 


\section{Abstract}

Gene-drive systems offer an important new avenue for spreading beneficial traits into wild populations. Their core components, Cas9 and guide RNA (gRNA), can either be linked within a single cassette (full gene drive, fGD) or provided in two separate elements (split gene drive, sGD) wherein the gRNA-bearing element drives in the presence of an independent static source of Cas9. We previously designed a system engineered to turn split into full gene drives. Here, we provide experimental proof-of-principle for such a convertible system inserted at the spo11 locus, which is recoded to restore gene function. In multigenerational cage studies, the reconstituted spo11 fGD cassette initially drives with slower kinetics than the unlinked sGD element (using the same Mendelian vasa-Cas9 source), but eventually reaches a similar level of final introgression. Different kinetic behaviors may result from transient fitness costs associated with individuals co-inheriting Cas9 and gRNA transgenes during the drive process. 


\section{Introduction}

CRISPR-based gene drives offer novel approaches for vector control by transforming genetic structures of wild insect populations ${ }^{1-5}$. Linked gene drives (or full gene drives, fGD) carry gene cassettes that encode the bacterial Cas9 endonuclease and a guide RNA (gRNA) sequence that directs the Cas9/gRNA ribonucleoprotein complex to cleave the genome at the site of cassette insertion ${ }^{3-5}$. Upon cleavage of the target site on a homologous chromosome, the gene-drive cassette is copied into the double stranded break (DSB) by homology-directed repair (HDR) using the drive-bearing homologous chromosome as a repair template. If this directional gene conversion process is efficient, the drive allele will be inherited in super-Mendelian fashion $(>50 \%)$. Genes encoding beneficial factors can be linked to fGD cassettes to spread these traits into susceptible populations within a few generations ${ }^{3}$.

Gene-drive technologies can be used either to modify or suppress insect disease vector populations depending on their design. Modification drives ${ }^{3,6,7}$ carry a beneficial cargo to reduce the vector capacity of the insect, while suppression drives ${ }^{5,8,9}$ endeavor to bias the inheritance of deleterious traits that will ultimately either kill or sterilize the insect. In addition to autonomously acting fGDs in which Cas9 and gRNA are encoded within a unitary cassette, socalled split gene-drives (sGD) have also been developed ${ }^{10-12}$ in which the copying cassette carries only the gRNA component, with Cas9 supplied from a second genomic site. In such bi-partite arrangements, the gRNA-carrying cassette is copied in the presence of the Cas9 source while the Cas9-encoding element is static (i.e., transmitted in a standard Mendelian fashion). In the absence of Cas9, however, the gRNA-bearing split-drive element also is inherited at Mendelian frequencies.

Because the copying component of a split gene drive spreads in an additive rather than exponential fashion ${ }^{4}$, these elements have been deemed safer and offer the means for more localized alterations of target populations ${ }^{13}$. The split configuration also provides greater flexibility for experimental analysis of the various CRISPR components including different promoters driving Cas9 expression and comparison of alternative gRNAs ${ }^{12,14}$. These advantages 
obviate the need for developing new full gene drives to test each pair of Cas9 and gRNA, which is time-consuming, expensive, and requires a higher level of biological confinement. A modification of the traditional split gene drive design is the trans-complementing gene drive (tGD) ${ }^{11}$, in which the gRNA cassette carries a second gRNA that targets cleavage of the genome at the site where the Cas9 element is inserted. When kept as separate stocks, the Cas9 and gRNA encoding elements are inherited in a Mendelian fashion, but when they are combined (e.g., by crossing stocks carrying the elements to each other) they behave as a conjoined full gene drive system. Although there are many advantages associated with the tGD system, it also is difficult to confine once assembled as a dual unit. Furthermore, the requirement of driving two separate elements magnifies the effect of drive-resistant mutations generated by the alternative nonhomologous end-joining (NHEJ) pathway ${ }^{15}$ that can arise at either gRNA cleavage site, potentially limiting the drive kinetics and final fraction of the population carrying both elements.

Our group recently analyzed a set of sGDs inserted into essential genes carrying functional, recoded cDNA sequences that restore functionality of the endogenous loci upon allelic cleavage and conversion ${ }^{12}$. Such recoded SGDs minimize the production of non-functional NHEJ alleles that are resistant to Cas 9 cleavage by dominantly eliminating such alleles transmitted by females through a process referred to as sterile/lethal mosaicism ${ }^{6,16}$. This latter process is based on maternal inheritance of Cas9/gRNA complexes that act on the paternal allele to mutate it in a sufficient fraction of cells in progeny to either kill (lethal mosaicism) or sterilize (sterile mosaicism) such individuals. Although unexploited in the previous study ${ }^{12}$, the sGD constructs included another design feature that permits facile genetic transfiguration of the SGD into an fGD. This conversion system relies on a set of Cas9 sources that carry sGD homology arms flanking the Cas9 cassette and a gRNA (gRNAHack) that can cleave a synthetic target site within the sGD element. When these Cas9 sources (inserted into AttP sites in different genomic locations) are crossed to the SGD element, the gRNAHack mediates cleavage of the SGD resulting in the insertion of the Cas9 cargo into the recipient element. Here, we provide proof-of-concept for such conversion of a recoded split drive into a full drive inserted into the spo11 locus, which is required for fertility in both sexes. This strategy for converting a SGD into a fGD follows the logic 
of the homology-assisted CRISPR knock-in system (or HACK) ${ }^{17}$, efficiently used to generate DNA double-stranded breaks and replace GAL4 cassettes in Drosophila. This flexible genetic swap system can rapidly convert an optimally performing SGD into an fGD and permits direct comparison of the performance of split versus full-drive systems that are inserted at identical genomic sites and are powered by the same promoter-Cas9 transgene. We evaluate the performance of a converted (or hacked) fGD in both single generation crosses and cage competition experiments and compare its performance to that of the SGD described in previous experiments ${ }^{12}$. We find that while the fGD displays slower initial drive kinetics, it catches up to the SGD and achieves a similar level of final introgression into the population. The delayed drive of the fGD element may result from a transient fitness cost we have previously described in SGD experiments that is associated with co-inheritance of Cas9 and gRNA transgenes in presence of cleavage-sensitive target alleles ${ }^{12}$. 


\section{$\underline{\text { Results }}$}

\section{Design of convertible SGD elements and corresponding Cas9 lines}

In previous single generation experiments, the recoded spo11 sGD line ${ }^{12}$ copied efficiently in females (76-83\% transmission) and to a lesser degree in males (65-70\% transmission), and drove efficiently to a high level of stable introduction in multi-generational cage experiments ( $\sim 5 \%$ ). Because this drive performed well but not perfectly, we selected it to test for conversion to an fGD by hacking (Figure 1a) since it would permit comparison of SGD versus fGD performance parameters in single generation crosses and in multi-generational cage trials. As a first step, we generated Cas9 donor lines, in which vasa or nanos (nos)-driven Cas9 transgenes are placed between a partial non-functional fragment of a tdTomato (tdTom, expressed in the eye) marker and a fully active EGFP transgene (expressed in the abdomen) that act as homology arms (HA) adjacent to a gRNA cleavage site in the sGD element (Figure 1b, spo11 line). As detailed in Figure $1 \mathrm{~b}$, the SGD carries a synthetically-designed PAM site (fauxPAM) between the two marker sequences, one of which is complete and active for the sGD line (tdTom) whereas the other one is partial and inactive (EGFP) and provides the recognition site for the hacking gRNA carried outside of the homology arms on the Cas9-donor element (Figure 1b, Hacked line). The donor Cas9 transgenes, endowed with the potential to hack sGDs and referred hereon as Cas9Hack, were inserted into available AttP sites located in either the II or III chromosomes via phiC31 recombination ${ }^{18}$ (Figure 1c). In addition to the aforementioned Cas9, HA, and attB sequences, Cas9Hack contains a gRNA (gRNAHack) that targets the fauxPAM site in the sGD line and mediates Cas9 insertion into the locus. gRNAHack sequences are located outside the HA-flanked Cas9 transgene, however, and thus are not copied into the $S G D$ recipient construct (Figure $1 \mathrm{~b}$ ). When the tdTom-marked SGD and EGFP-labeled donor Cas9 elements are combined, gRNAHackinduced cleavage of the SGD element can occur. Successful conversion leads to the insertion of the Cas9 transgene plus the 5' portion of the EGFP transgene directly upstream of the 3' portion of the EGFP transgene into the sGD, conferring a dual fluorescence phenotype on the "hacked" element (3XP3-tdTom + Opie2-EGFP; Figure 1b, Hacked line) and bestowing autonomous selfmobilizing capacity on the composite element. 


\section{a}

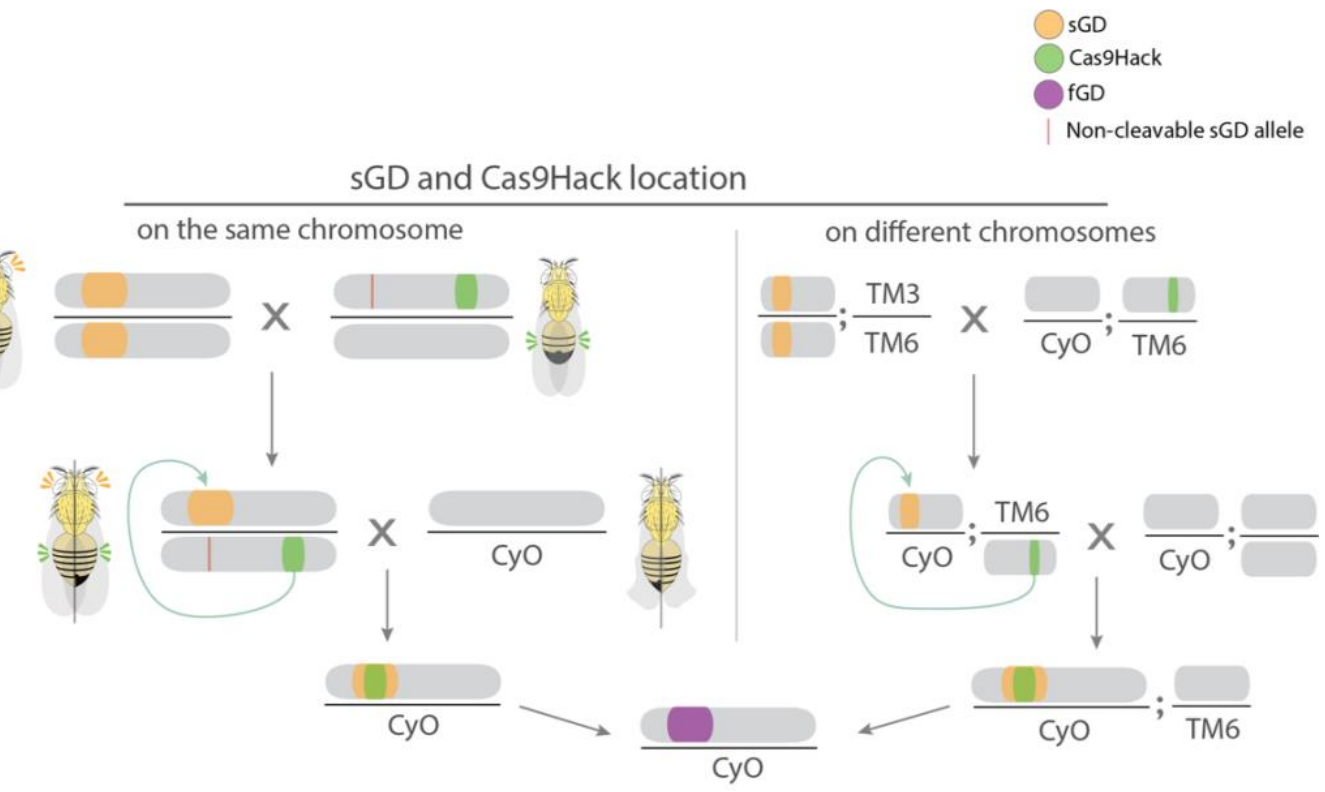

b

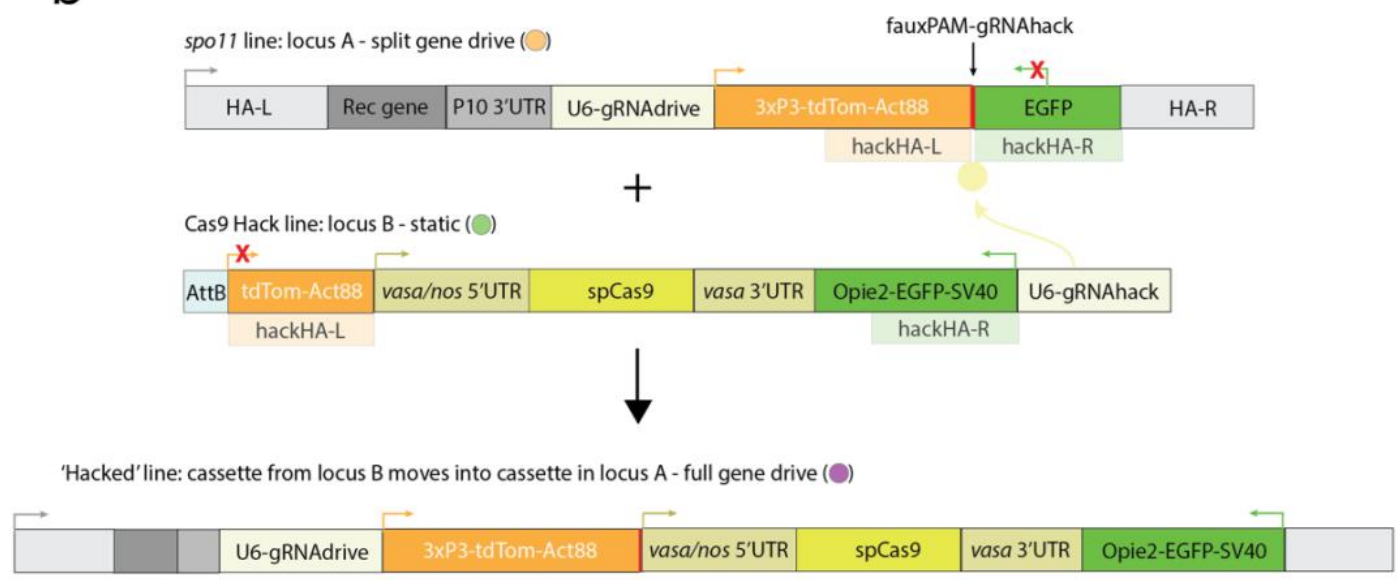

C

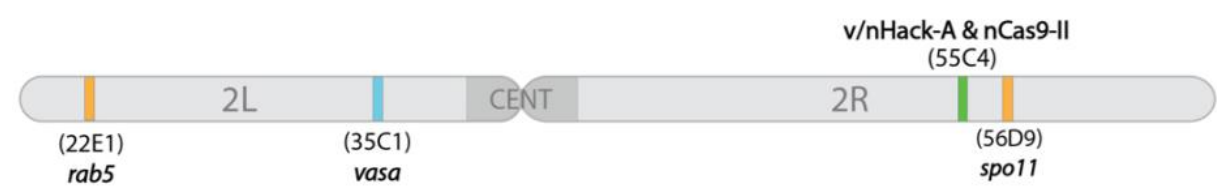

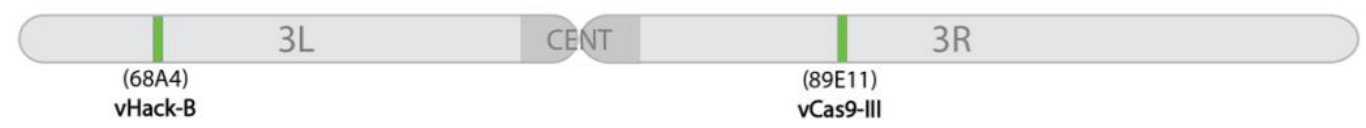

Figure 1 - Experimental design of the split to full gene drive conversion

a) Outline of the genetic cross schemes used to convert a split system into a full gene drive b) Schematic of the genetic constructs engineered and tested in the study for SGD to fGD conversion. A tdTom-expressing split gene drive cassette ( $S G D$ ) that contains a fauxPAM in between its markers is genetically paired with a specific EGFP-expressing Cas9, which contains a gRNA (that does not home) targeting the sequence next to the fauxPAM in SGD, that drives itself into the SGD locus and forms an autonomous full gene-drive cassette (fGD) using marker sequences as homology arms. The resulting cassette expresses both tdTom and EGFP markers c) Chromosomal location of the sGDs tested for conversion and hacking-Cas9 transgenes in the Drosophila melanogaster genome. 


\section{Cas9Hack and control Cas9 lines sustain comparable copying of a sGD inserted at the vasa locus}

In our prior study ${ }^{12}$, we employed two Cas9 sources (vCas9-III and nCas9-II) expressed under the control of different promoters (vasa and nos, respectively) to drive copying of the spo11 sGD. These two autosomal static Cas9 sources, inserted into chromosomes III and II, respectively, served as reference controls for assessing the activity of Cas9Hack sources controlled by the same promoters (vCas9Hack and nCas9Hack). The Cas9Hack transgenes were inserted in two genomic locations, 55C4 on chromosome arm 2R (Cas9Hack-A) and 64A4 on 3L (Cas9Hack-B).

In initial comparative experiments, we tested the newly generated Cas9Hack-A lines for their ability to support super-Mendelian inheritance of a reference unhackable mCerulean-marked split drive element inserted in the vasa locus on chromosome II (vCC; Figure 1c, Figure 2a). We chose not to use the fauxPAM-bearing spo11 sGD to test Cas9Hack lines to avoid complications arising from gRNAHack cutting the target element simultaneously with allelic conversion and copying of that element (via its own gRNA). Homozygous $\mathrm{G}_{0}$ virgin vCC females were mated to Cas9Hack males to obtain flies trans-heterozygous for both elements (Figure 2a). Single $F_{1}$ heterozygote males or virgin females were then crossed to wildtype flies (WT) of the opposite gender and their $\mathrm{F}_{2}$ progeny were scored for presence of the blue and green fluorescent markers carried by the vCC and Cas9Hack elements, respectively (Figure 2a). We observed comparable allelic conversion efficiencies for vCC using either the traditional vasa and nos-driven Cas9 (vCas9III and nCas9-II) sources and Cas9Hack transgenes when expressed using the corresponding promoters (vHack-A and nHack-A; Figure 2a). When allelic conversion occurred in females, both traditional Cas9 lines performed comparably (vCas9- $I I I=78 \pm 11 \%$; nCas9- $I I=77 \pm 5 \%$ ). Similar transmission values were also observed for Cas9Hack-A lines (vHack-A=78 $\pm 9 \%$; nHack-A $=82 \pm 10 \%$ ), which did not differ significantly from the control rates of vCas9-III and nCas9-IImediated copying (two-tailed Mann-Whitney test for vasa: $U=494.5, p=0.99$; nos: $U=170.5$, $p=0.07) . F_{2}$ progeny derived from heterozygous vCC/Cas9 males displayed somewhat lower conversion frequencies as previously observed for several split drive systems in Drosophila, which may be related to the anomalous absence of male recombination in this species ${ }^{11}$. Again, however, no significant differences were observed between Cas9 sources (vCas9-III=68 $\pm 8 \%$, 
nCas9-II=66 $\pm 7 \%$, vHack-A=67 $\pm 10 \%, n H a c k-A=68 \pm 10 \%$ ) (two-tailed Mann-Whitney test for vasa: $U=233, p=0.91$; nos: $U=195, p=0.94)$. In all cases, due to the static nature of the tested Cas9 sources, inheritance of the Cas9 transgenes approximated the expected Mendelian frequencies (average among all conditions: $51 \pm 8 \%$; Figure $2 \mathrm{a}$ ).

\section{Generation of spo11 fGD lines}

The comparable performance of Cas9Hack and reference Cas9 lines suggested that these transgene donor constructs were suitable for converting SGDs into fGDs by hacking. We crossed the hackable spo11 sGD with both vHack-A and nHack-A to obtain spo11 fGDs (Figure 1a). Of the 6 crosses we set up for each hacking element, we recovered double-marked/CyO individuals in $75 \%$ of them (5/6 for vHack-A, 4/6 for nHack-A). The predicted genomic structures of spo11 hacked cassettes were verified for fGD elements (hacked with vasa: vfGD or nos: nfGD) using PCR amplification followed by Sanger sequencing. In contrast to recovery of these multiple independent hacking events, an identical vHack construct inserted at a different III-chromosome AttP site (vHack-B) did not produce any successful recombination events, underscoring the

previously described role of genomic position effects on gene conversion efficiencies ${ }^{16}$ (Figure 1c). Since the spo11 sGD is closely linked to the vHack-A insertion site while the vHack-B locus is unlinked, the more efficient performance of vHack-A relative to vHack-B is consistent with prior observations of more efficient hacking from proximal loci and $\mathrm{Hi}-\mathrm{C}$ sequence analysis in which neighboring segments of the genome tend to lie closer together ${ }^{19}$. Consistent with this proximity hypothesis, the vHack-A (2R), which efficiently sustained hacking of the closely linked spo11 sGD did not do so when coupled with the more distant rab5 sGD (2L).

\section{spo11 sGD allelic conversion rates are slightly higher than for the unitary spo11 fGD element} Having successfully obtained several hacked spo11 fGDs carrying either the vasa-Cas9 or nosCas9 transgenes, we next compared their allelic conversion rates to those obtained with the bipartite spo11 sGD system. We crossed $G_{0}$ homozygote spo11 fGD males to homozygous WT virgin females and scored the fraction of fluorescently-labeled offspring (Figure $2 \mathrm{~b}$ ). In both sets of crosses, Cas9 was carried through males to avoid potentially confounding maternal 
transmission of the endonuclease through the female germline, although we note that attenuated carryover of this type could occur systemically in cells of fGD males due to inheritance from their grandmothers ${ }^{6,20}$. Emerging $F_{1}$ heterozygote flies were separated by gender, crossed with homozygous WT individuals of the opposite sex, and corresponding $F_{2}$ progeny were scored for presence of the red (tdTomato) and green (EGFP) eye fluorescent markers associated with the spo11 and Cas9 transgenes, respectively (Figure 2b). Paralleling our observations with the

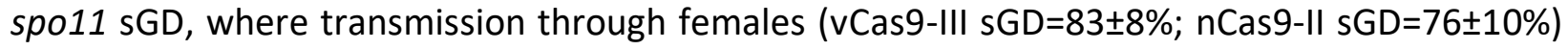
was greater than through males (vCas9-III sGD=65 $\pm 9 \%$; nCas9-II sGD=65 $\pm 10 \%$; Figure 2b), we detected gender-specific differences in both vfGD and nfGD. Overall transmission rates of fGDs from $F_{1}$ to $F_{2}$ progeny were approximately $75 \%$ (vfGD=75 $\pm 7 \%$, nfGD $=74 \pm 6 \%$; Figure $2 b$ ), slightly lower than those detected for the split SGD form in the case of the vCas9 source, but with comparable efficiencies observed between the fGD and sGD systems for nCas9. As mentioned above, transgene inheritance was substantially lower when passed through males, with only $\sim 60 \%$ of the progeny ( $\mathrm{vfGD}=60 \pm 6 \%, \mathrm{nfGD}=62 \pm 4 \%$; Figure $2 \mathrm{~b}$ ) receiving the transgene element, $a$ decrease of $5 \%$ relative to both the vCas9-III and nCas9-II SGD counterparts. These modest differences between SGD and fGD transmission efficiencies in both genders may reflect different sizes of the elements given that the fGD carries an additional $7 \mathrm{~kb}$ of cargo comprising Cas9 and its regulatory sequences. Low levels of residual Cas9/gRNA ribonucleoprotein complexes inherited from fGD grandmothers might also contribute to this slight attenuation. Alternatively, the chromosomal location of Cas9 transgenes may be relevant since Cas9 insertions on the third chromosome seemed to provide more efficient copying than those on the second chromosome. As expected, the static Cas9 sources were inherited at Mendelian frequencies in all of the spo11 sGD crosses (vCas9-III=52 $\pm 8 \%$ (both genders); nCas9- $I I=53 \pm 9 \%(F), 52 \pm 6 \%(M)$ ) whereas Cas9 remained linked to the spo11 element in the fGD (see super-Mendelian inheritance frequencies above; Figure $2 b)$. 

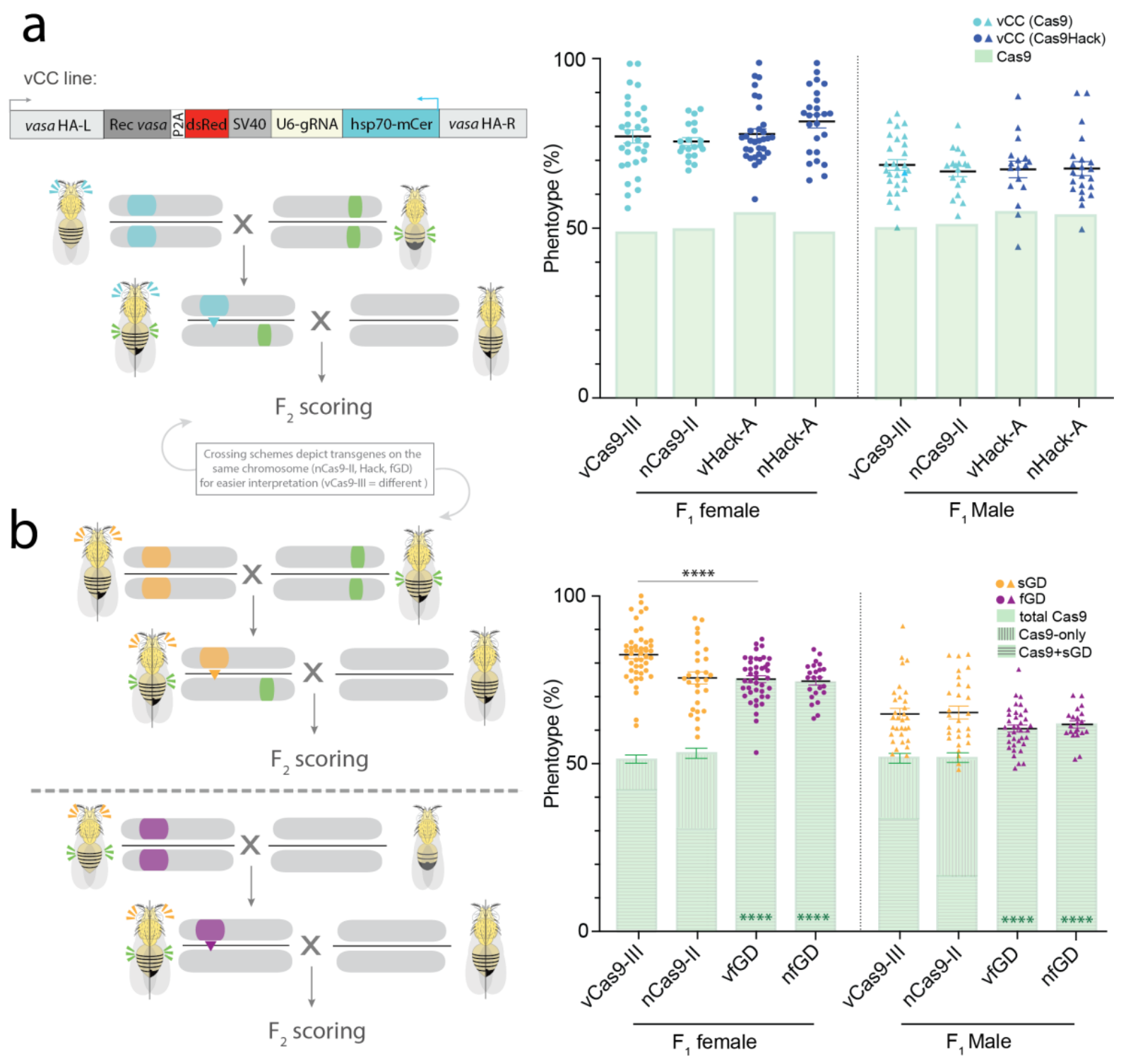

C single-pair crosses (NHEJ as heterozygotes)

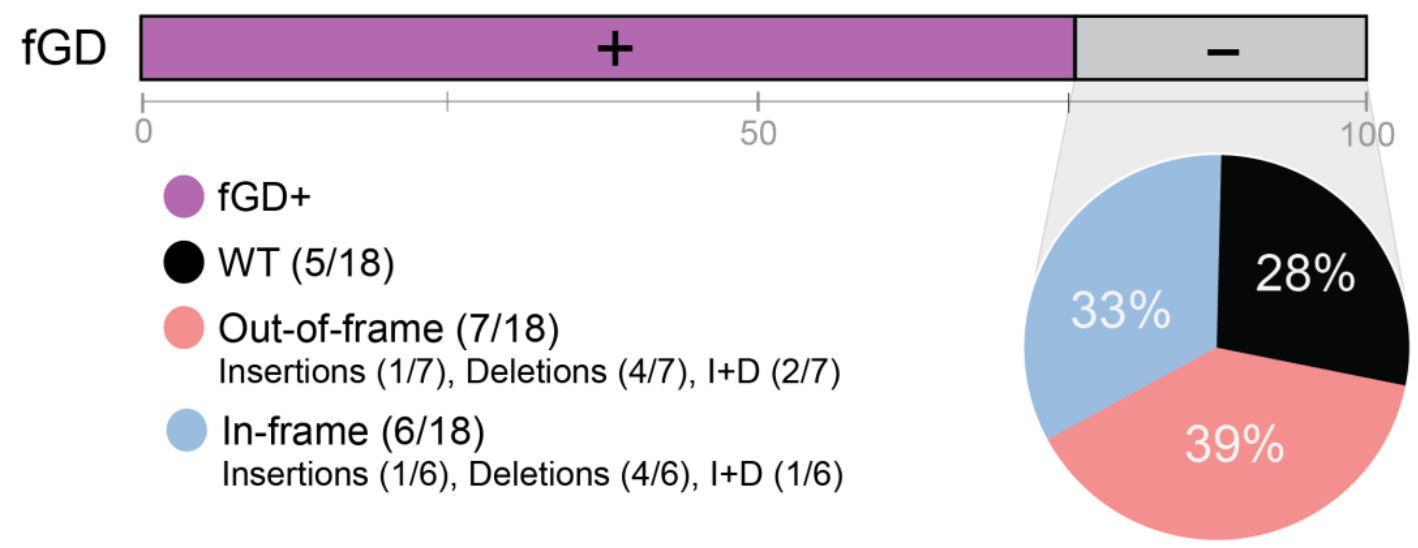


Figure 2 - Super-Mendelian performance is comparable between split and full gene-drive elements in singlegeneration crosses

a) Performance of hacking vasa and nos-driven Cas9 was tested and compared to previously used static Cas9 lines (all marked with EGFP) by crossing them to an unhackable split drive element (vCC) inserted in the endogenous vasa locus, marked with mCerulean. Single $F_{1}$ germline conversion was assessed by scoring the markers for both transgenes in the $\mathrm{F}_{2}$ progeny. Independent inheritance of Cas 9 and $\mathrm{VCC}$ is depicted using green bars and blue dots, respectively. b) Upon successful hacking of the spo11 sGD, vasa and nos-driven fGDs (vfGD and $n f G D$, respectively) were assessed for $F_{1}$ germline conversion capacities. The panel follows the layout described in a), except for the sGD marker being tdTomato and represented in orange and purple for sGD and fGD configurations, respectively. a, b) Sex of the parental $\left(F_{1}\right)$ trans-heterozygote is indicated in the $X$-axis under the Cas9 line used, as well as by circles (female) or triangles (male), used to show the data of each individual cross. Error bars represent mean values \pm SEM. Stars represent statistical significance $\left({ }^{* * *} p<0.0001\right)$ on sGD-copying differences (black, two-sided $t$-test) and Cas9 deriving from Mendelian frequencies (green, $\chi^{2}$ ). Raw phenotypical data is provided as Supplementary Data. c) Regions surrounding the spo11 gRNA target site were amplified from single non-fluorescent $F_{2}$ individuals generated in $b$ ), sequenced by Sanger and analyzed. A bar depicts the $\%$ of $\mathrm{fGD}^{+}$(purple) and \% of non-fluorescent (fGD', gray) flies. Genotypes obtained are depicted in a pie chart showing prevalence of indel mutations for the spo11 locus. Each section of the pie chart describes the kind of NHEJ allele that is formed and \% among the total tested $\mathrm{fGD}^{-}(\mathrm{NHEJ} / \mathrm{WT})$ heterozygotes.

As a complementary approach to assess fGD performance, we examined the in vivo cleavage efficiency of the spo11 fGD transgene by profiling the range of different NHEJ alleles generated in single-pair crosses (Figure $2 c$ ). We extracted DNA from non-fluorescent $F_{2}$ offspring (fGD ${ }^{-}$) derived from independent crosses of an $F_{1}$ trans-heterozygote female with a WT male. Following amplification and sequencing of the target sites in single $\mathrm{fGD}^{-} \mathrm{F}_{2}$ individuals, we estimated the frequencies of non-cleaved or NHEJ-induced alleles that were transmitted to the $F_{2}$ progeny from $F_{1}$ trans-heterozygous mothers by correcting for the father's WT allele from the Sanger sequencing reads. Analysis of 18 different $\mathrm{fGD}^{-}$flies revealed that $28 \%$ of the individuals inherited WT sequences, while 33\% carried in-frame indels, and 39\% had out-of-frame mutations (Figure 2c). Based on the sequencing data and super-Mendelian conversion frequencies observed in single crosses, we estimate the overall cleavage frequency for the spo11 fGD to be $\sim 93 \%$. This is very similar to the $92.3 \%$ cleavage frequency obtained previously for spo11 as a sGD ${ }^{12}$, confirming that cleavage efficiencies are locus-specific and not dependent on the size or nature of the transgenic cargo inserted at that locus. 
spo11 sGD and fGD differ in initial cage drive dynamics but not in final outcome

In prior studies, we paired the spo11 sGD with the vCas9-III source in cage trials seeded with initial ratios of $25 \%$ heterozygous (sGD/+; vCas9/+) to $75 \%$ WT $\left(+/+;+/+\right.$ ) individuals ${ }^{12}$. In those experiments, the frequency of the SGD increased rapidly to $70-85 \%$ introgression after 5-6 generations and then remained at a stable plateau due to the accumulation of non-deleterious (i.e., in-frame and presumably functional) NHEJ alleles (Figure 3a, orange traces). In these and other sGD experiments, we also observed that the static (or Mendelian) sources of Cas9 were eliminated from the population, which in the case of the spo11 sGD occurred after 15 generations (Figure 3a, green traces). Since Cas9 sources on their own or in combination with other sGDs did not disappear with similar kinetics, we inferred that their loss with certain sGDs (spo11 included) was due to fitness costs associated with carrying both sGD and Cas9 transgenes under conditions where uncleaved target alleles remained abundant.

Since transmission rates of $\mathrm{vfGD}$ and $\mathrm{nfGD}$ elements were comparable and similar to those achieved by the sGDs (albeit a bit lower) in single generation crosses, we selected vfGD for further comparison to vCas9-III SGD in multi-generational cage trials, where transgenic individuals face competition for mating and resources with WT individuals. We reasoned that these comparative experiments should provide insights regarding whether forced linkage of vCas9 to the gRNA within the same fGD cassette altered introduction of the fGD into a naïve population. Paralleling the sGD cage experiments, we seeded three replicate cages with $25 \%$ heterozygous (fGD/+) to 75\% WT (+/+) individuals (equally mature $\mathrm{G}_{0}$ males and $\mathrm{G}_{0}$ virgin females). Randomly-selected individuals were transferred at each generation for a total of 20 generations (Figure 3a). Following an initial rise in fGD frequency, we observed a gradual increase in the percentage of drive-bearing individuals in all cage replicates, which leveled off by approximately $\mathrm{G}_{12}$ (Figure $3 a$, purple traces). These fGD plateau frequencies remained stable over the remaining 8 generations and approximated those achieved by the SGD in prior experiments ${ }^{12}$. In the interval between the $\mathrm{G}_{4}$ and $\mathrm{G}_{10}$ generations, however, the drive trajectories of the SGD and fGD diverged significantly with the sGD increasing in frequency more rapidly than the fGD. 

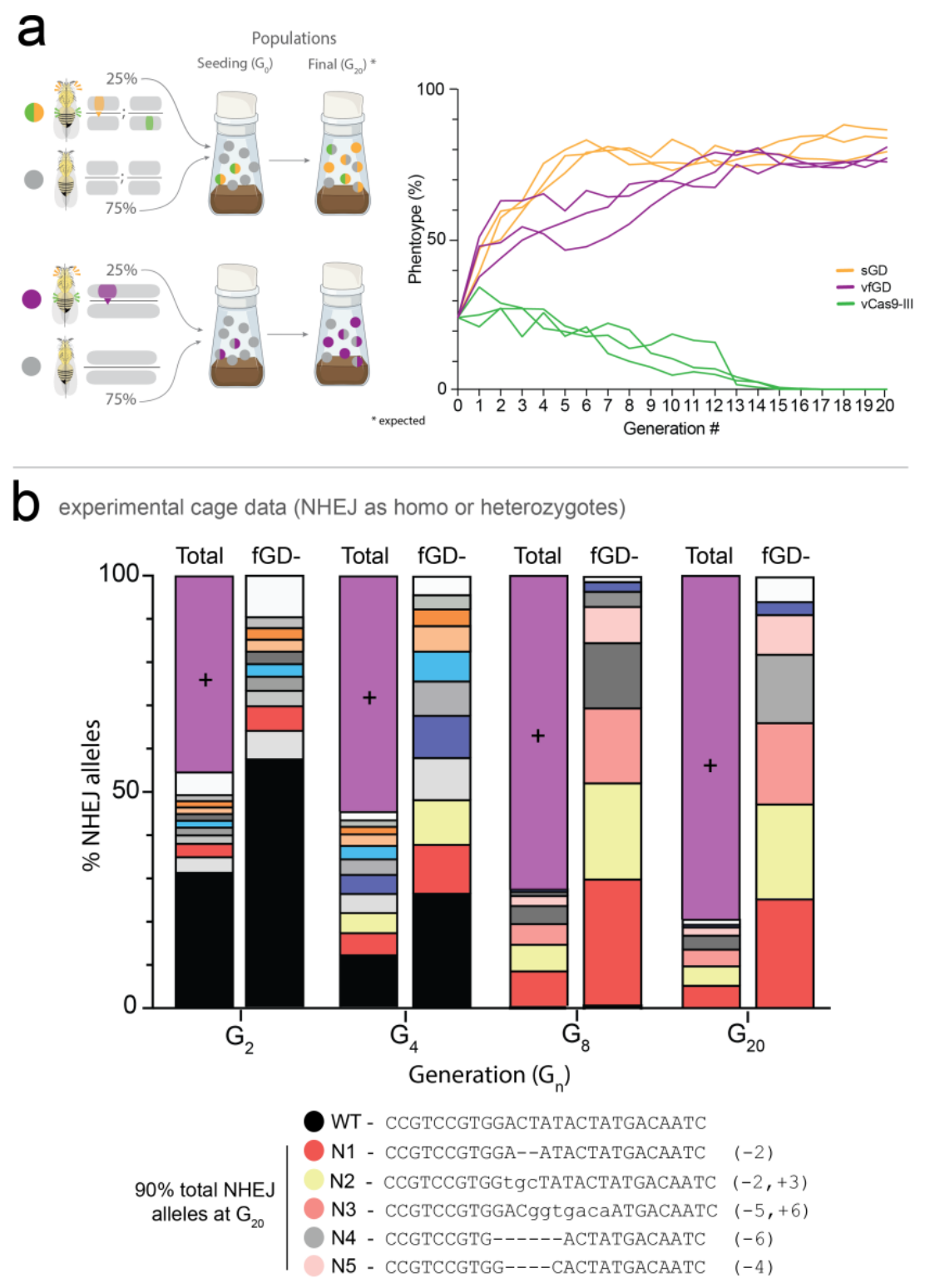

Figure 3 - spo11 fGD multi-generational cage trials and NHEJ profile assessment.

a) Setup of fGD cage trials followed previous experiments with the SGD-Cas9 configuration. Virgin heterozygote $\mathrm{fGD} /+$ and $W T(+/+)$ flies were seeded at 1:3 ratio in the initial generation and allowed to mate at random at each generation $\left(G_{n}\right)$. Flies in a cage were counted and scored for presence or absence of the phenotypic markers and randomly passed onto the following generation $\left(G_{n+1}\right)$. Orange and green traces depict sGD cage experiments where transgene and Cas9 are unlinked and sort independently of one another. Purple traces depict fGD cage trials, where transgene and Cas9 are linked as one genomic unit. b) NHEJ cage trial data was obtained by deep-sequencing the target site region of pooled non-fluorescent individuals at specific generations. Most prominent NHEJ alleles at each generation are shown in bars to represent their distribution among the total population ( $\mathrm{GGD}^{+}$and $\mathrm{fGD}$; left) or only in $\mathrm{fGD}^{-}$(right). Purple bars show the $\mathrm{fGD}^{+}$population percentage.

We also evaluated the DNA sequences of non-converted $\mathrm{fGD}^{-}$alleles in the population at four different timepoints: $\mathrm{G}_{2}, \mathrm{G}_{4}, \mathrm{G}_{8}$ and $\mathrm{G}_{20}$ (Figure 3 b) by sampling remaining non-converted alleles at every generation, then pooling $20-25 \mathrm{fGD}^{-}$individuals and performing deep-sequencing on the PCR-amplified regions flanking the Cas9 cut site. Sequence data revealed an array of different 
mutations at $\mathrm{G}_{2}$ and $\mathrm{G}_{4}$, but also large proportions of untouched WT alleles (Figure 3b, black bars). As was also the case of the sGD cage experiments ${ }^{12}$, we observed the progressive simplification of an initially complex array of mutations generated early during the drive process, most likely due to associated fitness costs. By $\mathrm{G}_{8}, \mathrm{WT}$ alleles were no longer detected, presumably because they had already been acted on by Cas9 carried by the fGD ${ }^{+}$allele and either successfully converted to carrying the $\mathrm{fGD}^{+}$element or mutated to cleavage-resistant and largely functional NHEJ alleles. As mentioned above, the variety of NHEJ alleles decreased over time leveling out by $\mathrm{G}_{8}$ and remaining stable through $\mathrm{G}_{20}$ (except for an allele represented in grey shading for which an extra $1 \mathrm{bp}$ deletion appeared between $\mathrm{G}_{8}$ and that of $\mathrm{G}_{20}$ ). Consistent with the disappearance of convertible WT alleles by $\mathrm{G}_{8}$ there was only a minimal increase in the frequency of the $\mathrm{fGD}^{+}$at $\mathrm{G}_{20}$.

\section{Mathematical models capture the major drive dynamics trends in population cages}

In our previous studies we modeled key drive parameters, including fertility fitness costs, for the spo11 sGD drive ${ }^{12}$. Building on those models, we extend our analysis to the fGD by assessing two potential fitness cost implementations: 1 ) an active cost associated with ascending phase of the drive trajectory, where fitness costs due to Cas9/gRNA were only apparent during active cleavage events, and 2) a co-occurrence cost, where the fitness costs manifest whenever an individual has Cas9 and gRNA, regardless of active cleavage. Drive performance was estimated from respective cage trial data using a Naive-Bayes Multi-Objective Hidden Markov Model optimized with an evolutionary algorithm (Figure 4a). Initial parameter estimates for transmission were taken from single-pair mating data (Figure $2 \mathrm{~b}$ ) and fitness costs from the previous study ${ }^{12}$, but with wider ranges to account for possible changes in fitness (see Mathematical Supplementary for a complete description of inheritance implementation, HMM, likelihood function, and estimated parameters). Cleavage rates approached 95\% (95\% quantiles: 90-95\%, see Mathematical Supplementary Tables S11 and S12), with significantly higher HDR in females (49\%, 95\% quantiles: $40-58 \%)$ than males (20\%, 95\% quantiles 20-37\%). Cleavage and conversion rates correspond to transmission estimates of $73 \%$ in females (95\% quantiles: $68-78 \%$ ) and $60 \%$ in males (95\% quantiles: 59-68\%), consistent with single-pair mating observations (Figure $2 b$ ). 
Rates of in-frame vs out-of-frame NHEJ events varied widely, but preferentially towards higher in-frame rates, consistent with our previous work and the importance of spo11 for fertility. However, the models lean towards no fitness cost from Cas9 and gRNAs, under either the active or co-occurrence models, which does not correlate with what was observed for the spo11 transgene acting as SGD. This limitation of the modeling can be explained by virtue of the hacked design; the initially separated fluorescent markers from the sGD and Cas9 constructs are now linked in a single cassette ( $f G D$ ), effectively presenting a single fluorescent marker than can only distinguish $\mathrm{GD}^{+}$versus $\mathrm{GD}^{-}$phenotypes. Thus, we applied the estimated fitness costs of Cas 9 and gRNAs for the spo11 transgene as sGD and obtained similar parameter estimates. Additionally, we used sequence data mentioned above from a sample $\mathrm{GD}^{-}$flies (Figure $3 c$ ) to estimate the $\mathrm{GD}^{-}$ allele frequencies (see SI File, Mathematical, section Data Preparation). When considering the modest amount of sequencing data relative to the phenotypic assessments ( $~ 80$ flies sequenced, compared to 19,386 flies counted for phenotype data), our models are heuristically equivalent to the measured allele frequencies with differences only minimally impacting our parameter estimates.

We generated stochastic realizations of our model, employing the active Cas9/gRNA cost and parameter estimates (see SI File, Mathematical, Tables S8 and S10, and Figure S1 for SGD and Tables S9 and S12, and Figure S3 for fGD), for comparison with the experimental cage data (Figure $4 \mathrm{~b}$ and $\mathrm{c})$. The stochastic model captured the potential role of chance events such as mate choice (multinomial-distributed), egg production (Poisson), progeny genotype (multinomial), and the finite sampling of the next generation (multivariate hypergeometric). Stochastic model trajectories were consistent with the cage trials but slightly below the long-term behavior of the sGDs (Figure 4b), likely due to the build-up of NHEJ alleles during the early generations in fGD cages corresponding to the drive delay. Additionally, allele frequencies (Figure 4c) indicate continued clearance of out-of-frame NHEJ alleles, consistent with winnowing deleterious alleles of an essential gene and with sequencing results obtained (Figure $3 \mathrm{~b}$ ). 

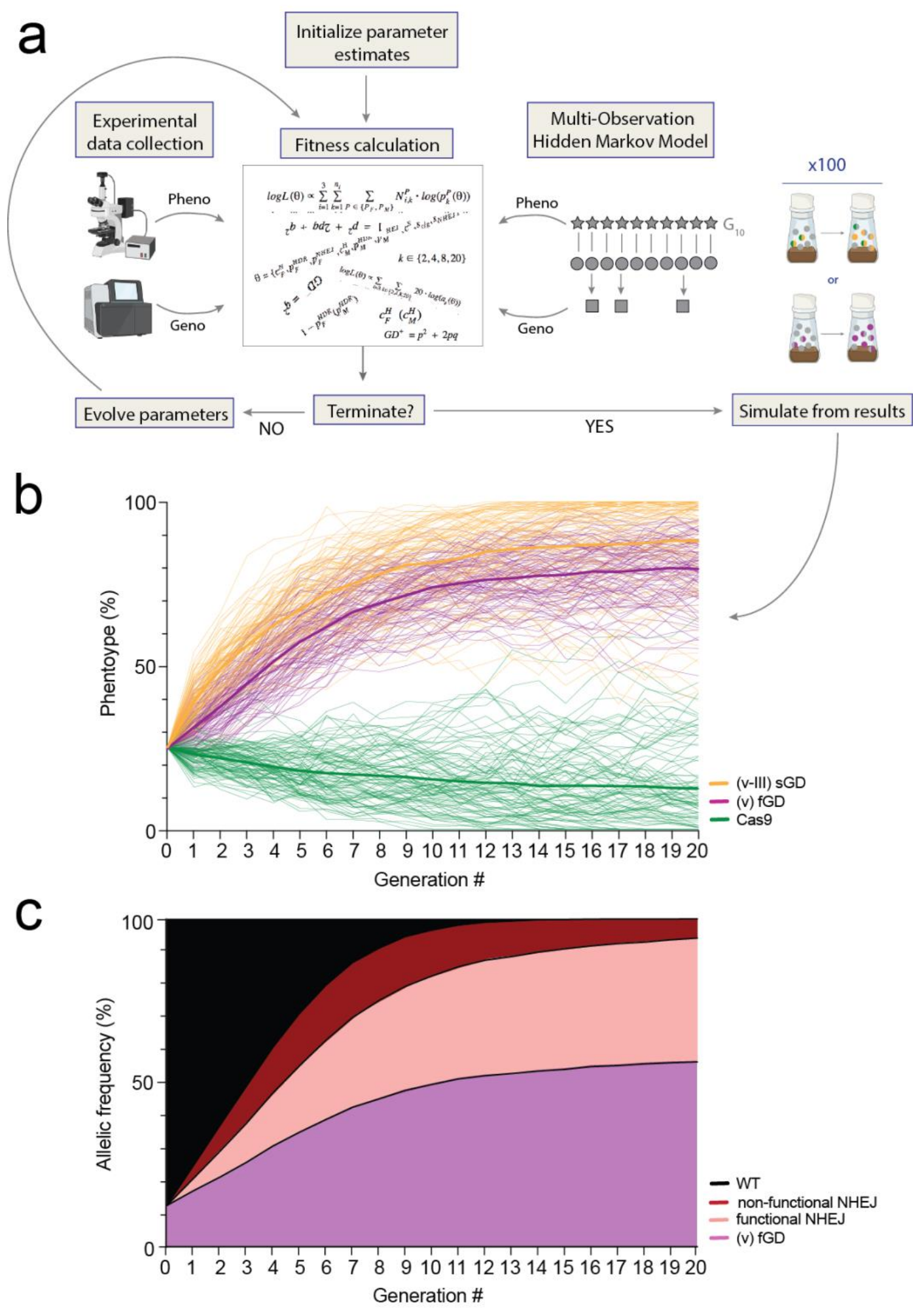

\section{Figure 4 - Mathematical model simulations provide insights in dynamics}

a) Schematic of the workflow to obtain accurate mathematical models for spo11's sGD and fGD configurations and their associated fitness parameters. b) Models were run using fitted parameter values and 100 stochastic simulations plotted for spo11 fGD (purple) and compared to spo11 sGD (orange for gRNA; green for Cas9). Thicker lines depict the mean of the 100 simulations. c) Predictions of alleles present in the fGD cage simulations at each generation. Purple, red shades and black curves depict $\mathrm{fGD}^{+}$, different types of NHEJ and WT allele, respectively. 


\section{Discussion}

In this study, we genetically converted a split gene-drive element targeting a locus essential for fertility in both sexes (spo11) into a full gene-drive. The sGD element was designed to contain homology arms flanking a Cas9 transgene that acts as a donor template for insertion into the sGD upon cleavage of the locus. We observed that sGD-to-fGD conversion efficiency depends greatly on the proximity between Cas9-Hack elements and target SGD loci. We tested two Cas9 transgenes (vHack-A or nHack-A driven by vasa or nos, respectively) that were closely linked (7$10 \mathrm{cM})$ to the SGD (2R) and assessed their capacity to copy into a suitable transgene located in the spo11 locus. Both vasa and nos Cas9Hack-A transgenes supported sGD-to-fGD conversion at high frequencies, as we observed multiple hacking events ( 3 of every 4 crosses). However, when testing the exact same hacking Cas9 donor constructs (CasHack-B) located on a different chromosome (3L) or an alternative freely recombining target SGD line ( $r a b 5)$ located in the opposite chromosome arm (2L) in combination with the vHack-A line homologous recombination events were not recovered. These observations agree with those described previously ${ }^{17,21,22}$, as chromosomal repair or HACK efficiency varied depending on genomic distance and orientation of the target-donor pair. Hi-C distances are highly relevant in this context, as they estimate the actual physical DNA proximity between two loci within meiotic nuclei. Thus, in future efforts to convert additional sGDs to fGDs, Cas9Hack lines inserted near the genomic integration site of the sGD element in question may prove most efficient.

Following successful recovery of both spo11 vfGD and nfGD lines, we assessed their copying performance in single generation crosses and compared them to that of their non-autonomous sGD counterparts. Paralleling outcomes observed with sGD crosses, all fGD-carrying females displayed a greater average conversion efficiency (75\%) than males (62\%) as has been generally observed for autosomal drives in Drosophila ${ }^{11,12,23}$, which may be related to the anomalous absence of male recombination in this species. In single generation crosses, the spo11 fGD lines copied at slightly lower rates ( $\sim-7 \%)$ than the sGD. The chromosomal position of the Cas9 transgene relative to spo11, or the greater overall size of the fGD element, may contribute to these modest reductions in conversion efficiencies. Regarding the potential role of genome 
position effects for Cas9 transgene insertion, we compared all Cas9 lines against a non-hackable $s G D, v C C$, and observed that the inheritance of the transgene was very similar for all of them. While chromosomal position of the tested Cas9 sources did not matter in this instance, Cas9dependent drive performance has been noted in other SGD-Cas9 combinations ${ }^{12,14}$. Cassette size may also influence conversion efficiencies, which could also be relevant in the context of the fGD element as the hacking mechanism inserts the whole promoter-Cas9 cassette into the locus, increasing the $f G D$ cassette size to $~ 8 k b$ longer than the $s G D$. Reduced copying efficiency has also been observed for larger drives inserted into the same yellow locus ${ }^{11,24}$.

We also tested the performance of the spo11 vfGD in multi-generational cage trials where we observed that it had the capacity to spread through a naïve population, as expected, following initial introduction at low levels. When seeded at a frequency of $12.5 \%$ of the total alleles (phenotypic: $25 \%$ ), the vfGD phenotype increased steadily to $80 \%$ of the population in 15 generations. This steady-state level corresponds to a similar degree of final introgression, but with delayed drive kinetics, compared to cage studies with the spo11 sGD paired with the corresponding vasa-Cas9 source. In that case, we observed a similar increase in total frequency, but in approximately half the number of generations ${ }^{12}$. Final levels of introgression were comparable for the two systems since they both plateau after reaching a phenotypic frequency of $75-85 \%$, mostly due to the generation of a similar number and complexity of non-deleterious resistant NHEJ alleles. In the previous study ${ }^{12}$, we observed that there was a moderate fitness cost associated with carrying certain SGDs and a Cas9 transgene in the same individual. In contrast to the SGD configuration, where copying of the gRNA-bearing cassette and static Cas9 transgene can separate during transmission (due to independent assortment of the Cas9 transgene), Cas9 is forcibly linked to gRNA in the fGD and thus a higher percentage of individuals will incur costs associated with co-inheritance of these CRISPR components. This effect is particularly pronounced in early generations when the great majority of non-transgenic individuals will be homozygous for the wildtype genotype and thus have fitness advantage over the transgenic line during the drive process. Since fitness costs associated with co-inheritance of gRNA and Cas 9 transgenes are confined to the active drive phase (due to a strong form of lethal 
or sterile mosaicism acting on wild-type paternal alleles), the fGD eventually achieves levels of introgression comparable to the SGD and then remains at stable levels in the population as nearly all remaining alleles are cleavage resistant (and most likely functional).

Mathematical modeling confirmed that many of the inferred drive parameters are similar for the SGD and fGD population cage experiments. Such modeling is rapidly becoming requisite in the gene drive toolbox, both for the analysis of experimental data ${ }^{24,25}$ and simulation of potential results to inform conservation ${ }^{26}$ and disease-elimination campaigns ${ }^{27,28}$. In this work, we demonstrate the application of a Naive-Bayes multi-observation HMM that optimizes the regular HMM in a highly-parallel framework by incorporating phenotypic and genotypic data. Simulations using our models (Figure 4) recapitulate what we observed in experimental data and provide us with ranges of plausible fitness cost values and parameters (Supplementary Information file, Mathematical).

In summary, this study provides a direct comparison between split and full drive configurations and validation of a general method for efficiently converting the one to the other by genetic means. These studies reveal unexpected differences in the kinetics between the split versus coupled drives in which the former displayed more rapid initial drive than the full drive (most likely due to a transient fitness cost of obligate co-inheritance of Cas9 and gRNA transgenes) but then reach comparable levels of final introgression. Thus, both systems offer potential advantages for field applications. The split system provides the potential for more localized and controllable spread of the CRISPR components while full gene drives could be implemented in contexts where long-lasting protection conferred by associated effector genes may be desired. 


\section{Materials and Methods}

\section{Plasmid construction}

All plasmids were cloned using standard recombinant DNA techniques. Plasmid and genomic DNA sequences were amplified using Q5 Hotstart Master Mix (New England Biolabs, Cat. \#M0494S) and Gibson assembled with NEBuilder HiFi DNA Assembly Master Mix (New England Biolabs, Cat. \# E2621). Resulting plasmids were transformed into NEB 5-alpha chemically-competent $E$. coli (New England Biolabs, Cat. \# C2987), isolated and sequenced. Primer sequences used for the creation of the different Cas9Hack plasmids can be found in the Supplementary File. Spo11 and rab5 sGD plasmid construction has been described previously ${ }^{12}$. Recoded cDNA fragments were designed by using non sub-optimal alternative codons from CRISPR cut site to gene's stop codon and synthesized as gBlocks ${ }^{\mathrm{TM}}$ (Integrated DNA Technologies). Codon usage was kept as similar as possible to that of the endogenous sequence.

\section{Microinjection of constructs}

Plasmids were purified using the PureLink Fast Low-endotoxin Maxi Plasmid Purification kit (ThermoFisher Scientific, Cat. \#A35895). All plasmids were sequenced prior to injection. Embryo injections were carried out at Rainbow Transgenic Flies, Inc. (http://www.rainbowgene.com). Each Cas9Hack construct was injected into AttP-harboring lines expressing integrase in the X chromosome (Bloomington \#R8621 and \#R8622). Injected embryos were received as $\mathrm{G}_{0}$ larvae, allowed to emerge and 3-4 females were intercrossed to 3-4 males. $\mathrm{G}_{1}$ progeny were screened for positive transgene marker (green body). All transgenic flies that displayed the marker were then balanced using Sco/CyO (for Cas9Hack-A, located on the II chromosome) or TM3/TM6 (Cas9Hack-B, III chromosome) and kept on a $w^{1118}$ background. Homozygous stocks were kept in absence of any balancer alleles or markers associated to the initial inserted line. Correct and complete transgene insertions in homozygous stocks were validated through PCR amplification and Sanger sequencing. 


\section{Fly genetics and crosses}

Fly stocks were kept and reared on regular cornmeal medium under standard conditions at 20$22{ }^{\circ} \mathrm{C}$ with a 12-hour day-night cycle. sGD and Cas9Hack stocks were kept separate in glass vials in an ACL-1 fly room, freezing the flies for $48 \mathrm{~h}$ prior to their discard. To assess Cas9Hack lines ability to drive, we genetically crossed vCC to each Cas9Hack line. To do so, individual transheterozygote $F_{1}$ males or virgin females were collected for each $G_{0}$ cross and crossed to a wildtype fly of the opposite gender. Single generation crosses were grown at $25^{\circ} \mathrm{C}$. Inheritance

of both gRNA (>50\%) and Cas9 ( 50\%) were calculated using the resulting $\mathrm{F}_{2}$ progeny by scoring the phenotypic markers associated to each transgenic cassette. Hacking efficiency experiments and all experiments performed after successful Cas9 introduction into the spo11 sGD locus, spo11 Hack lines were maintained in plastic vials in a contained ACL-2 insectary dedicated to Drosophila gene drive research. Used vials or samples were frozen for at least $48 \mathrm{~h}$ prior to their removal from the facility to be discarded or worked with, respectively.

\section{Multigenerational cage trials}

All population cage experiments were conducted at $25^{\circ} \mathrm{C}$ with a 12 -hour day-night cycle using $250 \mathrm{ml}$ bottles containing standard cornmeal medium. Crosses between homozygous $\mathrm{fGD}^{+}$and wildtype $\left(\mathrm{fGD}^{-}\right)$flies were carried out to obtain $\mathrm{F}_{1} \mathrm{fGD}^{+} / \mathrm{fGD}^{-}$heterozygotes, which were used to seed the initial generation. Wildtype or heterozygote males and virgin females were collected and separately matured for 3 to 5 days. Cages were seeded at a phenotypic frequency of 25\% $\mathrm{fGD}^{+}$heterozygotes (15 males, 15 females) to $75 \% \mathrm{fGD}^{-}$(45 males, 45 females). Upon transfer into fresh bottles for each generation, flies were allowed to mate and lay eggs for 3 days, then were removed from the cage $\left(G_{n}\right)$ and bottles were kept for 10 days to allow for the subsequent generation to develop to adulthood. Adult progeny $\left(G_{n+1}\right)$ was randomly separated into two pools and scored; one pool collected for sequencing analyses while the other was used to seed the following generation. If the two pools differed much phenotypically, frequencies were averaged in order to reduce variability and stochastic extremes. Continuous sampling and passage was carried out for 20 generations. 


\section{Molecular analysis of resistant alleles}

To extract fly genomic DNA for single fly resistant allele sequence analysis, single flies were squashed in lysis solution (10 mM Tris-Cl pH 8.2, $1 \mathrm{mM}$ EDTA, $25 \mathrm{mM} \mathrm{NaCl}$ and $0.2 \mathrm{mg} / \mathrm{ml}$ proteinase $\mathrm{K}$ ), incubated at $37^{\circ} \mathrm{C}$ for $30 \mathrm{~min}$ and deactivated at $95^{\circ} \mathrm{C}$ for $2 \mathrm{~min}$. After extraction, each sample was diluted in water and stored at $-20^{\circ} \mathrm{C}$ if needed. $1-2 \mu$ of each diluted DNA extraction was used as template for a $25 \mu$ PCR reaction that covered the flanking regions of the gRNA cut site, which were used to sequence the alleles. Sanger sequencing in individual nonfluorescent flies was performed at Genewiz, Inc. in San Diego, CA to obtain the single-cross NHEJ data. NHEJ allele sequences were obtained from Sanger chromatographs by isolating the WT sequence first and then annotating the remaining allelic sequence. For cage trials, 20-25 nonfluorescent flies were pooled and their DNA extracted at each sampling generation $\left(G_{2}, G_{4}, G_{8}\right.$ and $\mathrm{G}_{20}$ ). Target sequences were amplified using specific gene primers that also contained adapter sequences. Non-fragmented amplicons were sequenced using Illumina-based technology (2x250bp reads, Amplicon-EZ, Genewiz), with final data being delivered as FASTQ reads and aligned to a reference sequence for each gene of interest to detect indel formation. Primer sequences used for either single fly or cage trial deep sequencing analyses can be found in the Supplementary File.

\section{Mathematical modeling}

Model fitting was performed using a discrete-generation adaptation of the Mosquito Gene Drive Explorer (MGDrivE) ${ }^{29}$ software. A multi-objective HMM, using the log-likelihood as a score function, was optimized by evolutionary algorithm and used to generate parameter estimates with corresponding 95\% quantiles. Mendelian inheritance was assumed except under cooccurrence of the Cas9 and gRNA constructs, when the split-drive (and HACK) design allowed active cleavage of the target chromosome and the possibility of super-Mendelian inheritance. When cleavage occurred, a fraction of the cut alleles could be properly repaired via HDR, and the remaining cut alleles underwent NHEJ repair, generating in or out-of-frame resistant alleles. The effects of shadow-drive ${ }^{6,20}$, in which Cas9 protein is deposited in the embryo of a female individual who does not carry the Cas9 allele, but whose own mother does, were also 
accommodated. Fitness costs were implemented as fractional reductions in male and female fecundity, consistent with spo11 activity, and tested under active cleavage conditions as well as co-occurrence of Cas 9 and gRNAs. Additional details on the model implementation and likelihood function used in SGD and fGD model fitting can be found in the Supplementary Information. Phenotype and genotype mappings are provided in Tables S2 through S7. Fitness cost implementations, active vs co-occurrence, are shown in Tables S8 and S9. Parameter descriptions, estimates, and 95\% quantiles are provided in Tables S10 through S13. The resulting fits are visualized, with cage trial data, in Figures S1 through S4. Figures S5 and S6 analyze parameter estimate correlations while Figures S7 and S8 are partial rank correlation coefficient (PRCC) analyses.

Simulated model trajectories for Figure 4 were generated using a stochastic implementation of the discrete-generation model. At each generation, adult females mate with males, thereby obtaining a composite mated genotype (their own, and that of their mate) with mate choice following a multinomial distribution determined by adult male genotype frequencies, modified by mating efficacy. Egg production by mated adult females then follows a Poisson distribution, proportional to the genotype-specific lifetime fecundity of the adult female. Offspring genotype follows a multinomial distribution informed by the composite mated female genotype and the inheritance pattern of the gene drive system. Sex distribution of offspring follows a binomial distribution, assuming equal probability for each sex. Female and male adults from each generation are then sampled equally to seed the next generation, with sample size proportional to the average size of the cage trials at that generation, following a multivariate hypergeometric distribution. All simulations were performed and analyzed in $\mathrm{R}^{30}$.

\section{Figure generation and statistical analysis}

Initial graphs were generated using Prism 9 (v9.2, GraphPad Software Inc., San Diego, CA) and modified using Adobe Illustrator (v25.4.1, Adobe Inc., San Jose, CA) to visually fit the rest of the non-data figures featured in the paper. Figures $3 a$ and $4 a$ contain parts that were generated using BioRender. 


\section{Safety measures}

All research involving non-hackable gRNA cassettes/split gene drives was performed in glass vials in an ACL-1 facility, whereas research involving full gene drives (spo11 Hack lines) was conducted in plastic disposable vials in an ACL-2 facility, in accordance with the Institutional Biosafety Committee-approved protocol from the University of California San Diego. All vials were frozen for $48 \mathrm{~h}$ prior to autoclaving and discarding the flies. ACL-2 samples were also frozen for at least $48 \mathrm{~h}$ before their removal of the contained facility.

\section{Data and code availability}

Primers used for plasmid construction and sequencing (single and deep-sequencing experiments) can be found in Table S1. Full plasmid sequences can be found at the end of the manuscript's Supplementary Information File. Raw experimental data is also provided in this paper as a Supplementary Data file. All remaining experimental data is available from the authors upon request. Modeling information can be found as a Supplementary Method of this study in the Supplementary Information file. A version of MGDrivE was used for simulation modeling and is freely available from the MGDrive GitHub repository (https://marshalllab.github.io/MGDrivE/). Specific code can be obtained from the authors upon request.

\section{Funding}

Research was supported by a Paul G. Allen Frontiers Group Distinguished Investigators Award to E.B., NIH grant R01GM117321, and by gift from the Tata Trusts in India to TIGS-UCSD. J.B.B. and J.M.M. were supported by the DARPA Safe Genes Program Grant (HR0011-17-2-0047).

\section{Competing Interests}

E.B. has equity interests in Agragene Inc. and Synbal Inc., companies that may potentially benefit from the research results. E.B. also serves on the company's Board of Directors (Synbal) and Scientific Advisory Board (Synbal and Agragene). The terms of this arrangement have been reviewed and approved by the University of California, San Diego in accordance with its conflictof-interest policies. All other authors declare no competing interests. 


\section{References}

1 Burt, A. Site-specific selfish genes as tools for the control and genetic engineering of natural populations. Proc Biol Sci 270, 921-928, doi:10.1098/rspb.2002.2319 (2003).

2 Gantz, V. M. \& Bier, E. Genome editing. The mutagenic chain reaction: a method for converting heterozygous to homozygous mutations. Science 348, 442-444, doi:10.1126/science.aaa5945 (2015).

3 Gantz, V. M. et al. Highly efficient Cas9-mediated gene drive for population modification of the malaria vector mosquito Anopheles stephensi. Proceedings of the National Academy of Sciences of the United States of America 112, E6736-6743, doi:10.1073/pnas.1521077112 (2015).

4 Gantz, V. M. \& Bier, E. The dawn of active genetics. BioEssays : news and reviews in molecular, cellular and developmental biology 38, 50-63, doi:10.1002/bies.201500102 (2016).

5 Hammond, A. et al. A CRISPR-Cas9 gene drive system targeting female reproduction in the malaria mosquito vector Anopheles gambiae. Nature biotechnology 34, 78-83, doi:10.1038/nbt.3439 (2016).

6 Adolfi, A. et al. Efficient population modification gene-drive rescue system in the malaria mosquito Anopheles stephensi. Nature Communications 11, 5553, doi:10.1038/s41467020-19426-0 (2020).

7 Carballar-Lejarazú, R. et al. Next-generation gene drive for population modification of the malaria vector mosquito, Anopheles gambiae. Proceedings of the National Academy of Sciences, 202010214, doi:10.1073/pnas.2010214117 (2020).

8 Kyrou, K. et al. A CRISPR-Cas9 gene drive targeting doublesex causes complete population suppression in caged Anopheles gambiae mosquitoes. Nature biotechnology 36, 10621066, doi:10.1038/nbt.4245 (2018).

9 Simoni, A. et al. A male-biased sex-distorter gene drive for the human malaria vector Anopheles gambiae. Nature biotechnology, doi:10.1038/s41587-020-0508-1 (2020).

$10 \mathrm{Li}, \mathrm{M}$. et al. Development of a confinable gene drive system in the human disease vector Aedes aegypti. elife 9, doi:10.7554/elife.51701 (2020).

11 López Del Amo, V. et al. A transcomplementing gene drive provides a flexible platform for laboratory investigation and potential field deployment. Nature Communications 11, 352, doi:10.1038/s41467-019-13977-7 (2020).

12 Terradas, G. et al. Inherently confinable split-drive systems in Drosophila. Nature Communications 12, 1480, doi:10.1038/s41467-021-21771-7 (2021).

13 Akbari, O. S. et al. BIOSAFETY. Safeguarding gene drive experiments in the laboratory. Science 349, 927-929, doi:10.1126/science.aac7932 (2015).

14 Kandul, N. P. et al. Assessment of a Split Homing Based Gene Drive for Efficient Knockout of Multiple Genes. G3: Genes/Genomes/Genetics 10, 827-837, doi:10.1534/g3.119.400985 (2020).

15 Esvelt, K. M., Smidler, A. L., Catteruccia, F. \& Church, G. M. Concerning RNA-guided gene drives for the alteration of wild populations. elife 3, e03401, doi:10.7554/eLife.03401 (2014). 
16 Guichard, A. et al. Efficient allelic-drive in Drosophila. Nature Communications 10, 16401640, doi:10.1038/s41467-019-09694-w (2019).

17 Lin, C.-C. \& Potter, C. J. Editing Transgenic DNA Components by Inducible Gene Replacement in Drosophila melanogaster. Genetics 203, 1613, doi:10.1534/genetics.116.191783 (2016).

18 Groth, A. C., Fish, M., Nusse, R. \& Calos, M. P. Construction of transgenic Drosophila by using the site-specific integrase from phage phic31. Genetics 166, 1775-1782, doi:10.1534/genetics.166.4.1775 (2004).

19 Lieberman-Aiden, E. et al. Comprehensive Mapping of Long-Range Interactions Reveals Folding Principles of the Human Genome. Science 326, 289-293, doi:10.1126/science.1181369 (2009).

$20 \mathrm{Li}, \mathrm{Z}$. et al. CopyCatchers are versatile active genetic elements that detect and quantify inter-homolog somatic gene conversion. Nature Communications 12, 2625, doi:10.1038/s41467-021-22927-1 (2021).

21 Wang, R. W., Lee, C.-S. \& Haber, J. E. Position effects influencing intrachromosomal repair of a double-strand break in budding yeast. PloS one 12, e0180994-e0180994, doi:10.1371/journal.pone.0180994 (2017).

22 Lee, C. S. et al. Chromosome position determines the success of double-strand break repair. Proceedings of the National Academy of Sciences of the United States of America 113, E146-154, doi:10.1073/pnas.1523660113 (2016).

23 Champer, J. et al. Reducing resistance allele formation in CRISPR gene drive. Proceedings of the National Academy of Sciences of the United States of America 115, 5522-5527, doi:10.1073/pnas.1720354115 (2018).

$24 \mathrm{Xu}, \mathrm{X} .-\mathrm{R}$. S. et al. Active Genetic Neutralizing Elements for Halting or Deleting Gene Drives. Molecular Cell, doi:10.1016/j.molcel.2020.09.003 (2020).

25 Pham, T. B. et al. Experimental population modification of the malaria vector mosquito, Anopheles stephensi. PLoS genetics 15, e1008440, doi:10.1371/journal.pgen.1008440 (2019).

26 Godwin, J. et al. Rodent gene drives for conservation: opportunities and data needs. Proceedings of the Royal Society B: Biological Sciences 286, 20191606, doi:10.1098/rspb.2019.1606 (2019).

27 Carballar-Lejarazú, R. \& James, A. A. Population modification of Anopheline species to control malaria transmission. Pathogens and Global Health 111, 424-435, doi:10.1080/20477724.2018.1427192 (2017).

28 Long, K. C. et al. Core commitments for field trials of gen e drive organisms. Science 370, 1417, doi:10.1126/science.abd1908 (2020).

29 Sánchez C., H. M., Wu, S. L., Bennett, J. B. \& Marshall, J. M. MGDrivE: A modular simulation framework for the spread of gene drives through spatially explicit mosquito populations. Methods in Ecology and Evolution 11, 229-239, doi:10.1111/2041-210x.13318 (2020).

30 Team, R. C. R: A language and environment for statistical computing. (2017). 